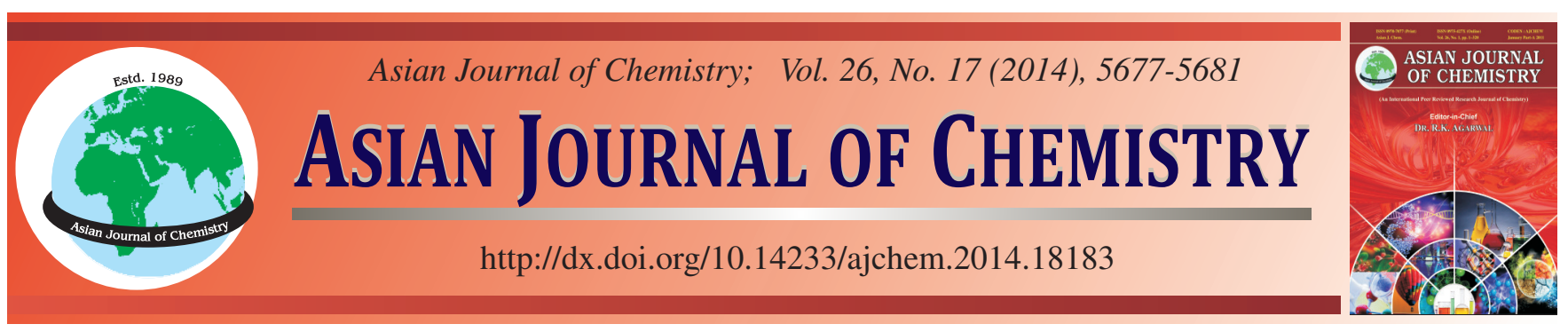

\title{
Speciation Characterization of Efficient Composite Inorganic Polymer Flocculant-Poly-ferric-zinc-silicate-sulphate $\dagger$
}

\author{
Y. LiU, S.Q. Li", S.M. LUO and W. WEI
}

School of Metallurgical and Ecological Engineering, University of Science and Technology Beijing, Beijing, P.R. China

*Corresponding author: Fax: +86 10 62332786; Tel: +86 10 82376226; E-mail: lisuqin@ metall.ustb.edu.cn

Flocculation process has been widely used in the water/wastewater treatment for many years. The continuous need for more efficient inorganic polymer flocculants has led to the development of newly modified flocculants, such as poly-ferric-zinc-silicate-sulphate (PFZSSB). The main purpose of this study is to examine the complexation of poly-ferric-zinc-silicate-sulphate by Ferron and Si-Mo timed spectrophotometric method and to provide a characterization of its structure and morphology by XRD and SEM. The experimental results indicated that poly-ferric-zinc-silicate-sulphate had a peculiar structure, being a new kind of polycrystalline compound, rather than a simple mixture of raw materials. The main existence form of polymeric Fe(III) species and silicate species were Fe(a) and $\mathrm{Si}(\mathrm{c})$, respectively. The introduction of $\mathrm{Zn}$ increased the degree of copolymerization among $\mathrm{Fe}, \mathrm{Si}$ and $\mathrm{Zn}$, leading to a colossal molecular structure and a complicated reticular system, which also made this flocculant far more active and stable than traditional ones.

Keywords: Inorganic polymer flocculants, Poly-ferric-zinc-silicate-sulphate, Flocculation.

\section{INTRODUCTION}

Flocculation is one of the important steps in the water/ wastewater treatment process. It is used for the destabilization of colloidal suspensions and the removal of suspended solids and organic matters ${ }^{1}$. During this process, inorganic flocculants have the widest range of applications because of their broad spectrum, low toxicity, low cost raw materials and good effects ${ }^{2,3}$. The traditional flocculants are aluminum and iron salts such as aluminum sulphate, ferric sulphate and ferric chloride ${ }^{4}$. The major drawbacks of these flocculants are that their treatment effect is not efficient enough so that the development and use of inorganic flocculant tends to increase molecular weight and the synergy among different components, so as to improve the flocculation effect ${ }^{5}$.

Over viewing the flocculants market in our country, inorganic polymer flocculants account for more than $8 \%$, such as polymeric ferric sulphate (PFS), polymeric ferric chloride (PFC) and poly aluminium chloride (PAC $)^{6-8}$. Generally, these flocculants carry high positive charge with high molecular weight, while the colloidal particles in the water/wastewater are almost negatively charged ${ }^{9}$. Therefore, when a certain flocculant is added into the water/wastewater, the charge neutralization mechanism makes these particles interact and forms larger flocs. However, their flocculation efficiency is still lower than that of the organic polymeric flocculants ${ }^{10,11}$.

Recently, a series of efficient composite inorganic polymer flocculants, which have exhibited higher flocculation efficiency than that of conventional polymeric ferric and aluminum flocculants in some situations, have been developed. They can break the colloid stability effectively and possess better adsorption and bridging functions, especially the poly-ferriczinc-silicate-sulphate (PFZSSB), which can leave out the introduction of certain organic flocculant (e.g. PAM). Moreover, it also has little limitation of water $\mathrm{pH}$. In the case of coking wastewater advanced treatment, the content of suspended solids could be decreased to less than $10 \mathrm{mg} / \mathrm{L}$, COD removal rate was above $50 \%^{12}$. As for the use in paper making wastewater advanced treatment, COD removal rate could reach above $70 \%$. Besides, it was also used in oily wastewater, beneficiation wastewater, etc., which showed an equal cost in comparison with the original process, but the result was better.

The hydrolytic polymerization morphology of $\mathrm{Fe}(\mathrm{III})$ along with its transforming rules is the key point of the study on ferric flocculants. Main research methods of the morphology of $\mathrm{Fe}(\mathrm{III})$ include chemical analysis, $\mathrm{pH}$ measurement, ultrafiltration, electron microscope, Ferron complexation timed spectrophotometric method, etc. ${ }^{13}$. Moreover, there has been 
a similar study based on a combined spectroscopic and titration techniques ${ }^{14}$. Above all, Ferron complexation timed spectrophotometric method is the most common one.

However, as a newly developed flocculant, interaction and morphology of various ingredients in PFZSSB has been studied inadequately, which limited the higher development of inorganic polymer flocculants. Therefore, PFZSSB was synthesized and characterized in the research. The purpose of this paper was to represent the complexation of PFZSSB by Ferron and Si-Mo timed spectrophotometric method and to determine the distribution of Fe(III) species and silicic species. Meanwhile, XRD and SEM were also used to analyze the structure and morphology of series of inorganic polymer flocculants e.g., PFSS, PFSSB, PFZSSB.

\section{EXPERIMENTAL}

Preparation of poly-ferric-zinc-silicate-sulphate: The efficient composite inorganic polymer flocculant PFZSSB was prepared by polymerization reaction, participating with sodium silicate $\left(\mathrm{Na}_{2} \mathrm{SiO}_{3} \cdot 9 \mathrm{H}_{2} \mathrm{O}\right)$, ferric sulphate $\left[\mathrm{Fe}_{2}\left(\mathrm{SO}_{4}\right)_{3} \cdot \mathrm{xH}_{2} \mathrm{O}\right]$, zinc sulphate $\left(\mathrm{ZnSO}_{4} \cdot 7 \mathrm{H}_{2} \mathrm{O}\right)$ and sodium tetraborate decahydrate $\left(\mathrm{Na}_{2} \mathrm{~B}_{4} \mathrm{O}_{7} \cdot 10 \mathrm{H}_{2} \mathrm{O}\right)$. All the reagents used were analytically pure.

The polysilicate acid solution was first prepared by dissolving $\mathrm{Na}_{2} \mathrm{SiO}_{3} \cdot 9 \mathrm{H}_{2} \mathrm{O}$ in distilled water. Then the solution above was regulated to a set acid $\mathrm{pH}$ with $4 \mathrm{~mol} / \mathrm{L} \mathrm{H}_{2} \mathrm{SO}_{4}$ and $2 \mathrm{~mol} / \mathrm{L}$ $\mathrm{NaOH}$ under rapid stirring, preventing a rapid gel at the neutral $\mathrm{pH}$. After a period of activation in the magnetic stirring apparatus, a set proportion of $\mathrm{Fe}_{2}\left(\mathrm{SO}_{4}\right)_{3} \cdot \mathrm{xH}_{2} \mathrm{O}, \mathrm{ZnSO}_{4} \cdot 7 \mathrm{H}_{2} \mathrm{O}$ and $\mathrm{Na}_{2} \mathrm{~B}_{4} \mathrm{O}_{7} \cdot 10 \mathrm{H}_{2} \mathrm{O}$ were added to the polysilicate acid solution and continually stirred it until fully reacted. The targeted flocculant, after being aged at least $24 \mathrm{~h}$, was analyzed to obtain its characterization and morphology.

The forming mechanism of PFZSSB was that the hydroxy in polysilicate acid and metal cation interacted and formed fractal-structure flocculant under certain conditions. There were a number of key factors that would affect the quality of flocculant, such as the molar ratio of metal ions and $\mathrm{SiO}_{2}$, excitation $\mathrm{pH}, \mathrm{SiO}_{2}$ mass percent concentration and so on. Therefore, the qualified PFZSSB could combine the advantages of polysilicic acid and metal salts and have a more superior flocculating performance than any single constituent.

Ferron complexation timed spectrophotometric method: The Ferron complexation timed spectrophotometric method was used to determine the distribution of Fe(III) species according to that the Ferron reagent can form different complexes with single iron ions, as well as other hydroxo complexes at all levels with an increase of reaction time ${ }^{15,16}$. This method can classify the aqueous species of Fe(III) into three different extents of hydrolysis and polymerization-Fe(a), $\mathrm{Fe}(\mathrm{b}), \mathrm{Fe}(\mathrm{c})$, respectively. $\mathrm{Fe}(\mathrm{a})$ represented the simple monomeric and oligomeric species that reacted almost instantly with Ferron. $\mathrm{Fe}(\mathrm{b})$ meant the transitional low polymeric species that reacted with Ferron gradually. Fe(c) consisted of more stable high polymeric species that did not react with Ferron ${ }^{17}$. As the Ferron solution was acidulous, the best condition of determination $\mathrm{pH}$ was around $4-5^{18}$.

Si-Mo complexation timed spectrophotometric method: The Si-Mo complexation timed spectrophotometric method was used to determine the distribution of silicic species, which were classified into three categories the same as Ferron method above, according to the discrepancy of reaction rates between different forms of silicon oxide and ammonium molybdate ${ }^{19-21}$.

X-Ray diffraction: Samples of solid powder PFZSSB were characterized by X-ray diffraction (XRD) for the determination of crystalline phases with X-ray diffractometer (D/MAX$\mathrm{RB}$, Japan) with $\mathrm{Cu} \mathrm{K}$ radiation in the $2 \theta$ at scan rate of $1 \%$ $\min ^{22}$. The solid PFZSSB was obtained by aging the liquid products for 10 days and then placing them into a homeothermic drying oven to dry for $100 \mathrm{~h}$. Besides, the solid samples of PFSS and PFSSB were also made to be characterized by $\mathrm{XRD}$ at the same conditions to make comparisons.

Scanning electron microscope: The 10-day-aged samples of PFSS, PFSSB and PFZSSB were dropped to the conducting resin (to be thin as far as possible) bonded on the plate and then dried at room temperature. Before being placed under the scanning electron microscope (SUPRA ${ }^{\mathrm{TM}} 55$, Germany), the plate should be sprayed by carbon to promote the conductivity.

\section{RESULTS AND DISCUSSION}

Determination of polymeric Fe(III) species: The study has tested three samples (PFSS, PFSSB, PFZSSB) with Ferron complexation timed spectrophotometric method and the content of $\mathrm{Fe}(\mathrm{a}), \mathrm{Fe}(\mathrm{b})$ and $\mathrm{Fe}(\mathrm{c})$, was calculated, respectively.

Results (Fig. 1) obtained from the Ferron assay showed that the quantity of $\mathrm{Fe}(\mathrm{a})$ accounted the largest part (above 60 $\%$ ) within all the three flocculants, $\mathrm{Fe}(\mathrm{c})$ came to the second, while $\mathrm{Fe}(\mathrm{b})$ was the lowest, which meant that it was inactive for $\mathrm{Fe}(\mathrm{III})$ species to translate from $\mathrm{Fe}$ (a) to $\mathrm{Fe}(\mathrm{c})$. Moreover, the reaction rate was low. Related research ${ }^{23}$ also suggested that there would be a slow response for Fe(III) species bonding with polysilicate acid to Ferron reagent. Therefore it could be reckoned that ferric ions were absorbed by polysilicate acid, leading to that the transformation from oligomeric species to high polymeric species was relatively slow and there was little quantity of $\mathrm{Fe}(\mathrm{b})$. By comparison, the content of $\mathrm{Fe}(\mathrm{b})$ and $\mathrm{Fe}(\mathrm{c})$ in PFSSB was more than that in PFSS, which indicated that the introduction of boron strengthened the connection between $\mathrm{Fe}^{3+}$, including its hydrolytic polymerization product and polysilicate acid colloidal particles, as well as $\mathrm{SO}_{4}{ }^{2-}$, thus making the reacting dose of $\mathrm{Fe}^{3+}$ increased.

In addition, the introduction of $\mathrm{Zn}^{2+}$ also contributed to the low content of $\mathrm{Fe}(\mathrm{b})$. The reason for this situation may be that the introduced $\mathrm{Zn}^{2+}$ would reinforce the polymerization of $\mathrm{Fe}, \mathrm{Si}$ and $\mathrm{Zn}$, making it difficult for the $\mathrm{Fe}$ (III) of polymer to react with Ferron reagent. On the other hand, with more $\mathrm{Fe}(\mathrm{b})$ in PFZSSB transforming to high polymeric species, the bridging and trapping function of PFZSSB was also enhanced. Overall, the content of $\mathrm{Fe}(\mathrm{c})$ increased gradually from PFSS to PFZSSB, meaning that high polymeric Fe(III) species had an increasing proportion in the polymerization, thus making the composite flocculant PFZSSB own a more macromolecular structure and high degree of polymerization, which resulted to the high efficient ability of PFZSSB.

Distribution of silicic species in PFZSSB: The eligible samples of PFZSSB were analyzed through the Si-Mo complexation timed spectrophotometric method at different aging 


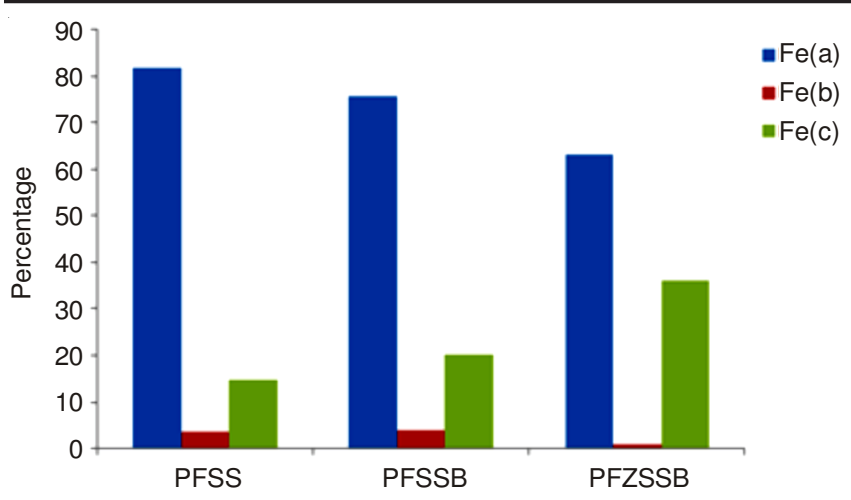

Fig. 1. Distribution of ferric species in different flocculants

time and $\mathrm{pH}$. The results for PFZSSB, which were summarized in Fig. 2, showed that there was over $50 \%$ of Si(c), constituting the largest part of all silicic species, which meant that high polymeric silicon accounted for the most in PFZSSB. Related research ${ }^{24}$ had illustrated that the characteristics of Si(a) and $\mathrm{Si}(\mathrm{b})$ were similar and had more activity than $\mathrm{Si}(\mathrm{c})$.

By contrast, there were more $\mathrm{Si}(\mathrm{c})$ and less $\mathrm{Si}(\mathrm{a})$ and $\mathrm{Si}(\mathrm{b})$ with an increase of aging time. That indicated that there was large amount of high polymeric silicon with higher molecular weight, making it easier to generate gelatinization. While the decrease of $\mathrm{Si}$ (a) meant that there was a transformation from $\mathrm{Si}(\mathrm{a})$ to $\mathrm{Si}(\mathrm{b})$ and finally to $\mathrm{Si}(\mathrm{c})$. Furthermore, it could be seen from Fig. 2(B) that the lowest content of $\mathrm{Si}(\mathrm{b})$ was only about $2 \%$, far more less than its counterparts, indicating that the transformation rate between $\mathrm{Si}(\mathrm{b})$ and $\mathrm{Si}(\mathrm{c})$ was much quicker than that between $\mathrm{Si}(\mathrm{a})$ and $\mathrm{Si}(\mathrm{b})$.

Besides, $\mathrm{pH}$ had a more significant effect on the silicic species of PFZSSB. Fig. 2 showed that there was a similar distribution of silicic species when $\mathrm{pH}$ was 2 and 3 , in which much more oligomeric species existed, making this flocculant more active and stable. While when the $\mathrm{pH}$ was 1 or 4 , the content of $\mathrm{Si}(\mathrm{c})$ was much more than that in 2 or 3 . In this situation $(\mathrm{pH}=1,4)$, the silicic acid formed high polymers that had very slow reaction rate. The reason for this may be that it would be easier for silicic acid to translate into the higher species at a strong acidic condition. On the other hand, the proportion of $\mathrm{Si}(\mathrm{a})$ and $\mathrm{Si}(\mathrm{b})$ decreased significantly when the $\mathrm{pH}$ rose to 4 while the $\mathrm{Si}(\mathrm{c})$ had an enormous percentage of over $80 \%$. This may be caused by the gelatinization of polysilicate acid and finally the flocculant became gel and out of action.

Overall, PFZSSB would have better flocculation performance and better stability when the $\mathrm{pH}$ was at $2-3$. It was mainly because that there were more oligomeric species at this situation, which could exist longer and would not transform into high polymers soon. $\mathrm{Zn}^{2+}$ also had some inhibition to the gelatinization of polysilicate acid at the same time.

Comparison between silicic species in different flocculants: In order to compare the silicic species of different flocculants, the research tested PSi, PFSS, PFSSB, PFZSSB with Si-Mo method at different aging time. The results illustrated in Fig. 3 showed that there tended to be fewer species of $\mathrm{Si}(\mathrm{a})$ and $\mathrm{Si}(\mathrm{b})$ and more $\mathrm{Si}(\mathrm{c})$.

Obviously, the content of $\mathrm{Si}(\mathrm{c})$ in PSi was too little that there was not enough macromolecule to conduct bridging and
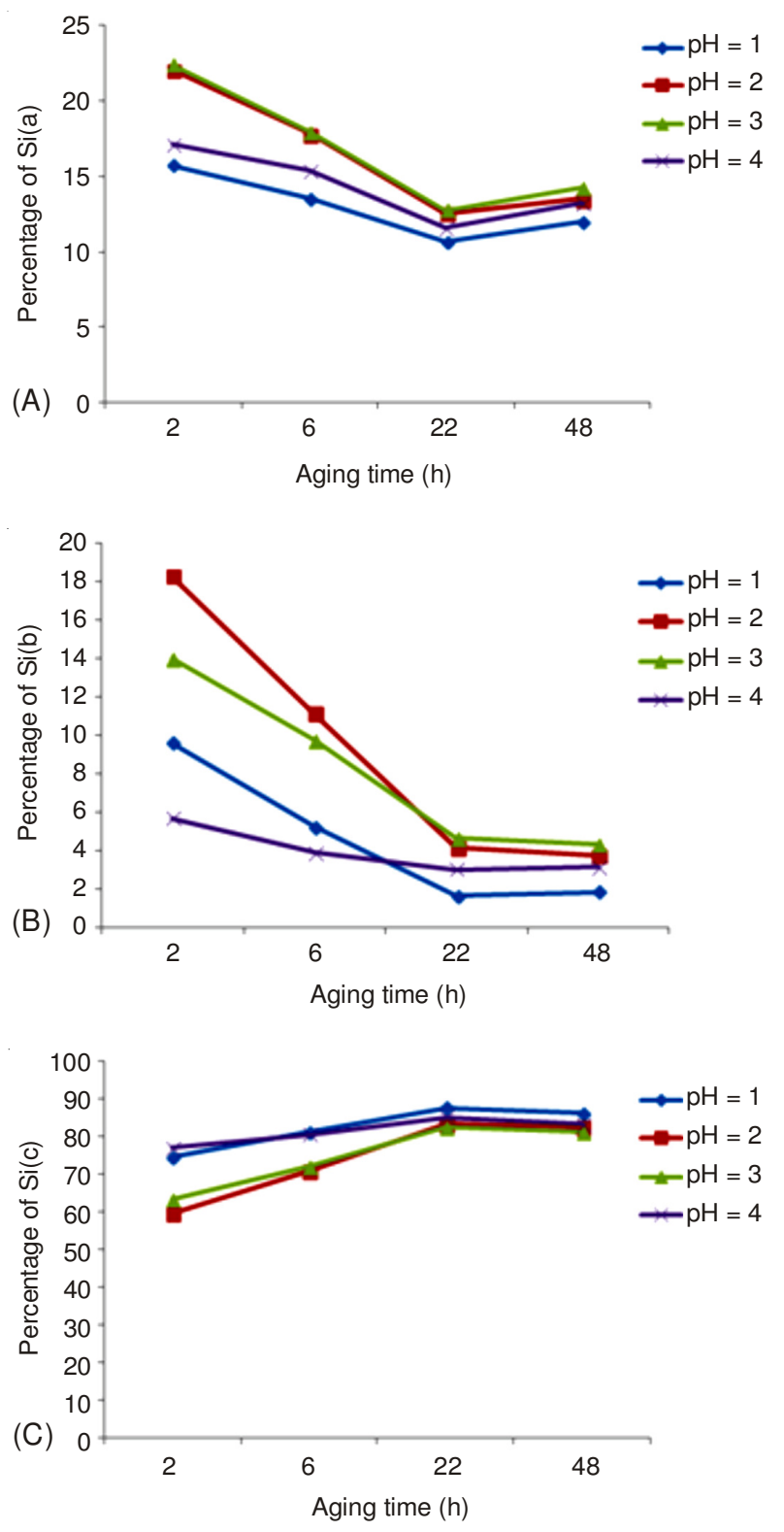

Fig. 2. Distribution of silicic species at different aging time and $\mathrm{pH}$

meshing functions, which also explained the poor result of PSi dealing with waste water. By contrast with PSi, PFSS had a more efficient distribution of silicic species. With the injection of iron ions, the proportion of Si(c) in PFSS increased, indicating that certain chelation existed between iron ions and silicic acid, which also resulted in the decrease of more oligomeric species. And there was also a downward trend in the content of $\mathrm{Si}(\mathrm{c})$ from the $22^{\text {nd }}$ aging hour to the $48^{\text {th }}$, which also illustrated that ferric ion exerted its inhibition function at the process of siliceous polymerization.

The boron in sodium tetraborate had an electron deficiency structure, which could combine with hydroxide radical, making itself a superior cross-linking agent. The injection of boron improved the stability of PFSS, which could be inferred in terms of the decrease of $\mathrm{Si}(\mathrm{c})$ between the $22^{\text {nd }}$ aging hour and the $48^{\text {th }}$ aging. There was a kind of balance between silicic acid and metallic ion during this period, slowing down the gelatinization of polysilicate acid. In the acidic region, certain bonding effects existed between boron and siliceous particles, 

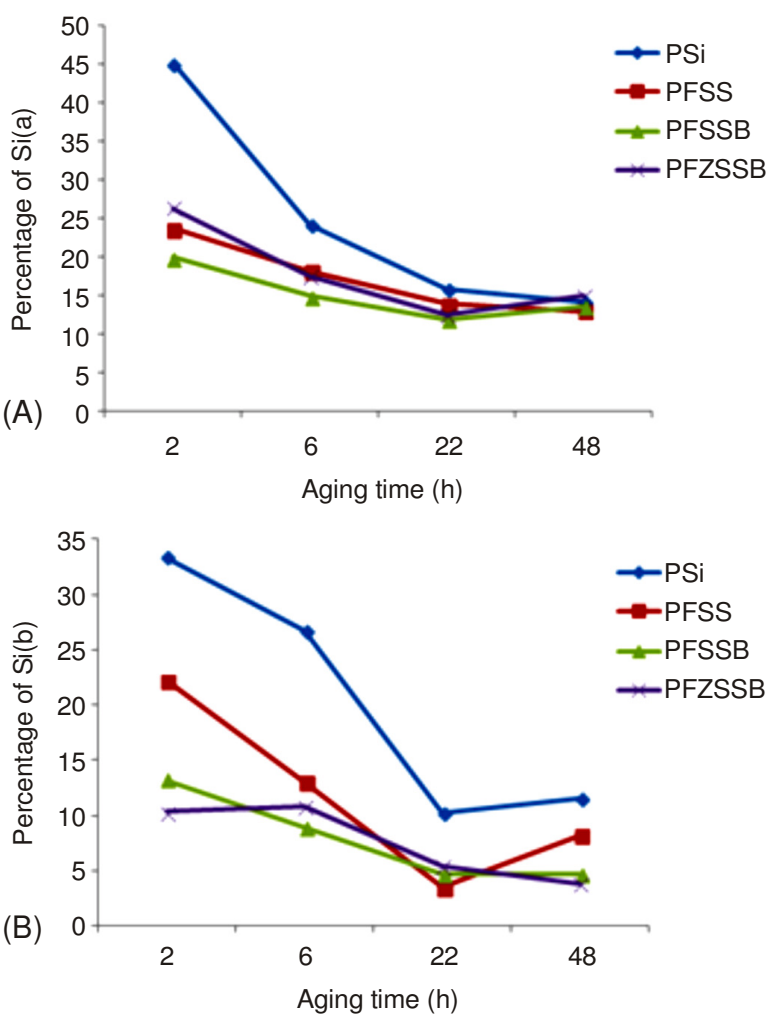

(A)
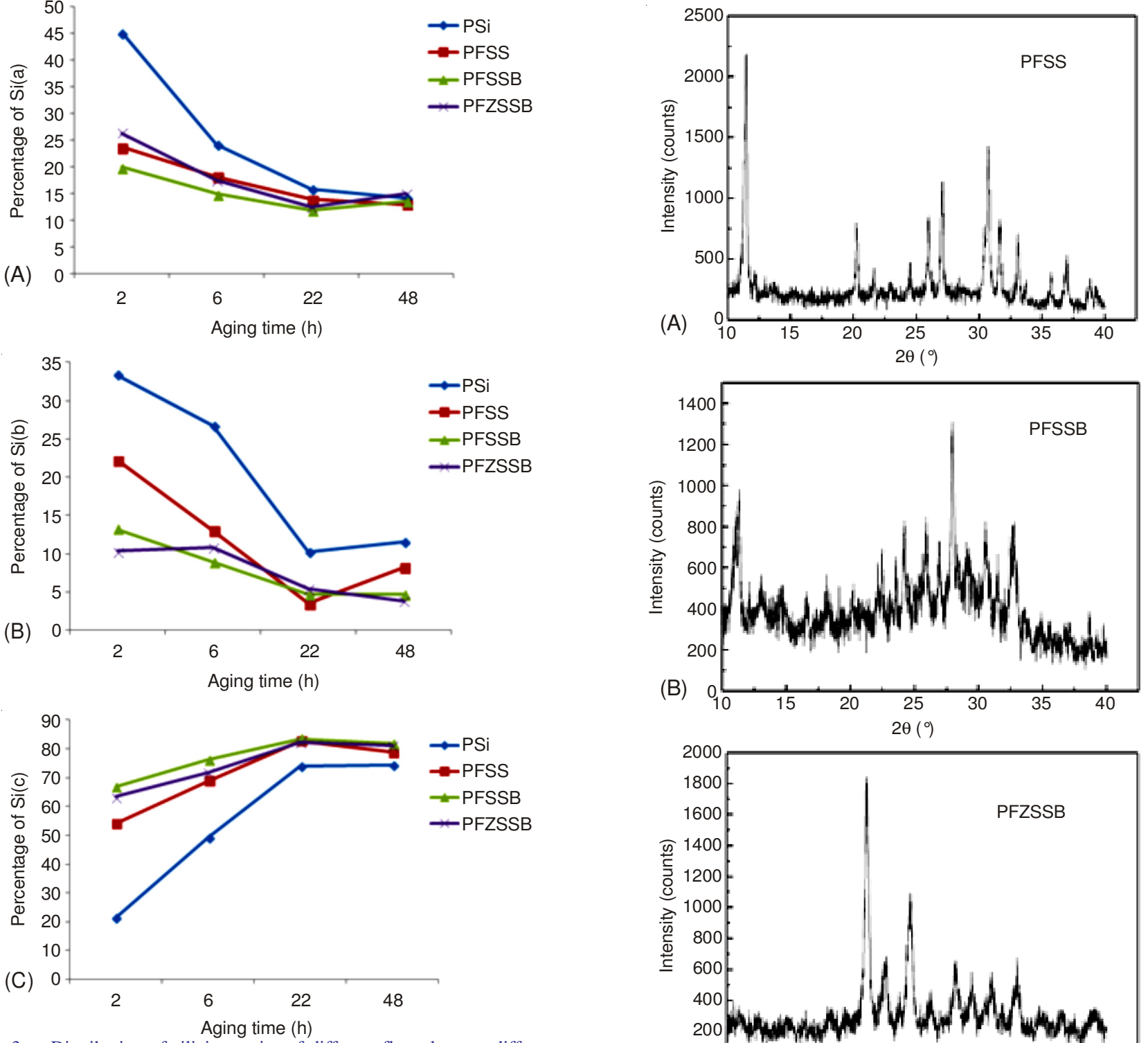

Fig. 3. Distribution of silicic species of different flocculants at different ageing time

forming the Si-O-B structure and hindering the further polymerization. At the same time, some $\mathrm{H}_{3} \mathrm{BO}_{3}$ was produced. $\mathrm{H}_{3} \mathrm{BO}_{3}$ reacted with not only the silicon hydroxyl in the surface of siliceous particles, but also hydroxide radical in the hydrolysate of metallic ion. This reaction composed chelates. As a result, the flocculants that contained boron had a higher proportion of $\mathrm{Si}(\mathrm{c})$. And when the aging time extended, the increasing rate of $\mathrm{Si}(\mathrm{c})$ was slower, ensuring the high effectiveness of the flocculants, as well as their stability.

The comparison between PFSSB and PFZSSB further indicated that the presented $\mathrm{Zn}^{2+}$ had an inhibition function in this process. Meanwhile, PFZSSB also had almost all the merits of PFSSB.

X-Ray diffraction analysis: Fig. 4 illustrated the XRD patterns of PFSS, PFSSB, PFZSSB. As shown in Fig. 4, all the three flocculants had largely amorphous structures with small traces of crystallinity and very little reactants can be identified. It was suggested that some new kind of polycrystalline compounds had been made, rather than a simple mixture of raw materials.

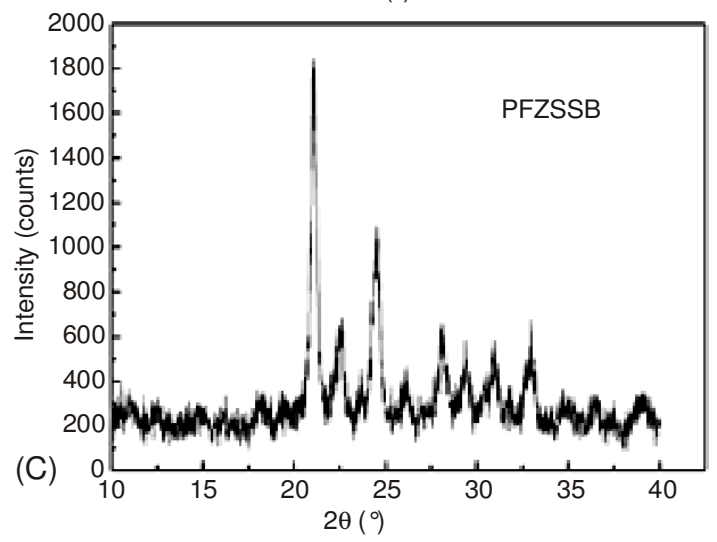

Fig. 4. XRD patterns of PFSS(A), PFSSB(B), PFZSSB(C)

The amorphous structures of PFSS indicated that polysilicic acid chelate and absorb $\mathrm{Fe}^{3+}$; while the picture of PFSSB showed that there was a kind of polymerization between polysilicic acid and boron. For the PFZSSB, whose peak shape was also in amorphous state, presented that diffraction peaks of ferric sulphate, zinc sulphate and sodium tetraborate, did not exist. In other words, the reaction was participated by $\mathrm{Fe}^{3+}, \mathrm{SO}_{4}{ }^{2-}, \mathrm{Zn}^{2+}$ and boron, which formed new amorphous polymers with polysilicic acid.

Scanning electron microscope analysis: The SEM microphotographs of PFSS, PFSSB, PFZSSB, were illustrated in Figs. 5-7. Fig. 5 showed that there were some branches in PFSS. By magnifying parts of the branches, a stratified structure could be observed. But more floc and thicker branches existing in the PFSSB (Fig. 6), confirmed that introduced boron had a significant effect on the flocculant. Fig. 7 presented a colossal molecular structure made of connected branches, which was almost full of the whole picture. This structure could not only 


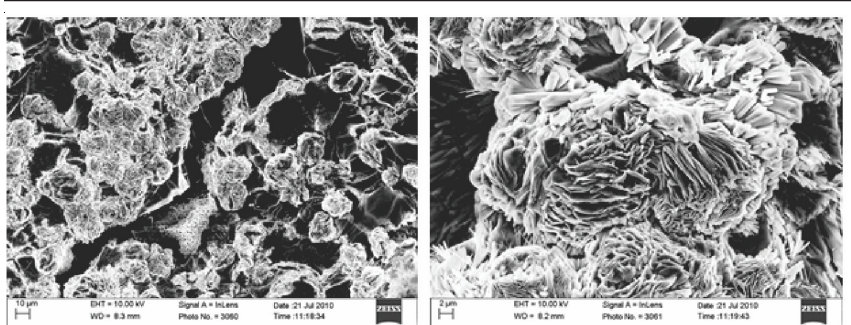

Fig. 5. SEM microphotograph of PFSS

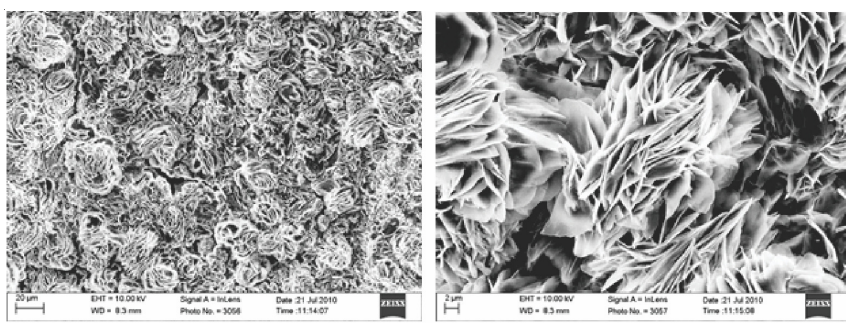

Fig. 6. SEM microphotograph of PFSSB
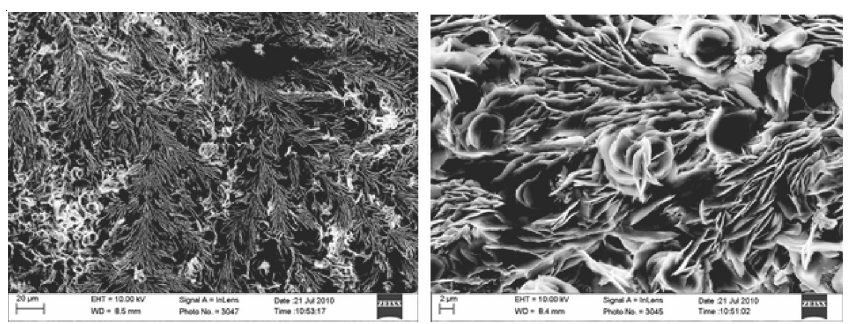

Fig. 7. SEM microphotograph of PFZSSB

contact with pollutants in the sewage, but also increase its flocculation effect and stability.

It is suggested that $\mathrm{Zn}^{2+}$ was prone to polymerize with polysilicic acid to form mesh and stratified structure and the introduction of $\mathrm{Fe}, \mathrm{Zn}$ and boron changed the morphology of polysilicic acid and increased its stability. Consequently, their interactive effects generated three-dimensional macromolecular polymers with various valence bond structure, which owned good adsorption properties and macromolecular bridging abilities.

\section{Conclusion}

In this paper, the distributions of polymeric $\mathrm{Fe}(\mathrm{III})$ and silicate species of PFZSSB were investigated, as well as its structure and morphology. From the above investigation, we came to the following conclusions:

- The study of Ferron complexation timed spectrophotometric indicated that $\mathrm{Fe}(\mathrm{III})$ species mainly existed in the form of simple monomeric and oligomeric species.

- The introduction of $\mathrm{Zn}$ increased the degree of copolymerization among $\mathrm{Fe}, \mathrm{Si}$ and $\mathrm{Zn}$, leading more $\mathrm{Fe}^{3+}$ to transforming to high polymeric species and enhancing the bridging and trapping function of PFZSSB.
- The study of Si-Mo complexation timed spectrophotometric showed that the main existence form of silicate species was $\mathrm{Si}(\mathrm{c})$ and it changed with aging time and activated $\mathrm{pH}$; PFZSSB would have better flocculation performance and better stability when the activated $\mathrm{pH}$ was 2-3.

- XRD analysis showed that PFZSSB had a peculiar structure; the inexistence of the diffraction peaks of ferric sulphate, zinc sulphate and sodium tetraborate indicated that a new kind of polycrystalline compound was formed in the process. SEM analysis demonstrated that under the combined action of $\mathrm{Fe}, \mathrm{Zn}$ and boron, PFZSSB possessed a colossal molecular structure and a complicated reticular system, leading to better bridging and meshing functions.

\section{REFERENCES}

1. A.I. Zouboulis and G. Traskas, J. Chem. Technol. Biotechnol., 80, 1136 (2005).

2. H.X. Tang, Z.K. Luan, D.S. Wang et al., In eds.: H.H. Hahn, E. Hoffman and H. Odegaard, Chemical Water and Wastewater Treatment V, Springer-Verlag, Berlin, Germany, Ch. 3, pp. 25-34 (1998).

3. D.S. Wang, H.X. Tang and F.C. Cao, Colloids Surf. A, 166, 27 (2000).

4. G.C. Zhu, H.L. Zheng, Z. Zhang, T. Tshukudu, P. Zhang and X. Xiang, Chem. Eng. J., 178, 50 (2011).

5. B.Y. Gao, Q.Y. Yue, B.J. Wang and Y.B. Chu, Colloids Surf. A, 229, 121 (2003).

6. N. Parthasarathy and J. Buffle, Water Res., 19, 25 (1985).

7. J.Q. Jiang and N.J.D. Graham, J. Chem. Technol. Biotechnol., 73, 351 (1998).

8. W.P. Cheng, Chemosphere, 47, 963 (2002).

9. J. Duan and J. Gregory, Adv.Colloid. Interf. Sci., 100, 475 (2003).

10. D.F. Zeng, J.J. Wu, J.F. Kennedy, Carbohydr. Polym., 71, 135 (2008).

11. J.P. Wang, Y.Z. Chen, S.J. Zhang and H.-Q. Yu, Bioresour. Technol., 99, 3397 (2008).

12. J. Zhou and S.Q. Li, Industrial Water Treatment, 28, 35 (2008).

13. C.M. Flynn Jr., Chem. Rev., 84, 31 (1984).

14. Aquilanti, M. Giorgetti, M. Minicucci, G. Papini, M. Pellei, M. Tegoni, A. Trasatti and C. Santini, Dalton Trans., 40, 2764 (2011).

15. A.I. Zouboulis, P.A. Moussas and F. Vasilakou, J. Hazard. Mater, 155, 459 (2008)

16. J.Q. Jiang and N.J.D. Graham, J. Chem. Technol. Biotechnol., 73, 351 (1998).

17. B.Z. Tian and H.X. Tang, Environ. Chem., 8, 27 (1989).

18. H.X. Tang, B.Z. Tian, Z.K. Luan and Y. Zhang, in eds.: H. Hahn and R. Klute, Inorganic Polymer Flocculant Polyferric Chloride, Its Properties, Efficiency and Production; In: Chemical Water and Wastewater Treatment, Springer-Verlag, Berlin, Germany, Vol. 3, pp. 57-69 (1994).

19. D.S. Wang and H.X. Tang, Environ. Chem., 16, 515 (1997).

20. J. Shi, Y. Zhang, K.Y. Zou and F. Xiao, J. Environ. Sci. (China), 23, 749 (2011).

21. D.S. Wang and H.X. Tang, Water Res., 35, 3418 (2001).

22. Y.B. Zeng and J. Park, Colloids Surf. A Physicochem. Eng. Asp., 334, 147 (2009).

23. Y.H. Song, X.Y. Yue and B.Y. Gao, Environ. Prot. Oil Gas Fields, 7, 4 (1997).

24. D.S. Wang and H.X. Tang, Chinese J. Environ. Sci., 22, 94 (2001) 